\title{
Spiking Noise and Information Density of Neurons in Visual Area V2 of Infant Monkeys
}

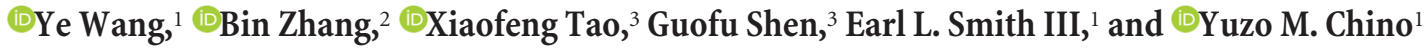 \\ ${ }^{1}$ College of Optometry, University of Houston, Houston, Texas $77204,{ }^{2}$ College of Optometry, NOVA Southeastern University, Fort Lauderdale, Florida \\ 33314, and ${ }^{3}$ Department of Ophthalmology, Baylor College of Medicine, Houston, Texas 77030
}

Encoding of visual information requires precisely timed spiking activity in the network of cortical neurons; irregular spiking can interfere with information processing especially for low-contrast images. The vision of newborn infants is impoverished. An infant's contrast sensitivity is low and the ability to discriminate complex stimuli is poor. The neural mechanisms that limit the visual capacities of infants are a matter of debate. Here we asked whether noisy spiking and/or crude information processing in visual cortex limit infant vision. Since neurons beyond the primary visual cortex $(\mathrm{V} 1)$ have rarely been studied in neonates or infants, we focused on the firing pattern of neurons in visual area V2, the earliest extrastriate visual area of both male and female macaque monkeys (Maccaca mulatta). For eight stimulus contrasts ranging from $0 \%$ to $80 \%$, we analyzed spiking irregularity by calculating the square of the coefficient of variation $\left(\mathrm{CV}^{2}\right)$ in interspike intervals, the trial-to-trial fluctuation in spiking (Fano factor), and the amount of information on contrast conveyed by each spiking (information density). While the contrast sensitivity of infant neurons was reduced as expected, spiking noise, both the magnitude of spiking irregularity and the trial-to-trial fluctuations, was much lower in the spike trains of infant V2 neurons compared with those of adults. However, information density for $\mathrm{V} 2$ neurons was significantly lower in infants. Our results suggest that poor contrast sensitivity combined with lower information density of extrastriate neurons, despite their lower spiking noise, may limit behaviorally determined contrast sensitivity soon after birth.

Key words: Fano factor; interspike interval; mutual information; noise; V2

Significance Statement

Despite $>50$ years of investigations on the postnatal development of the primary visual cortex (V1), cortical mechanisms that may limit infant vision are still unclear. We investigated the quality and strength of neuronal firing in primate visual area $\mathrm{V} 2$ by analyzing contrast sensitivity, spiking variability, and the amount of information on contrast conveyed by each action potential (information density). Here we demonstrate that the firing rate, contrast sensitivity, and dynamic range of V2 neurons were depressed in infants compared with adults. Although spiking noise was less, information density was lower in infant V2. Impoverished neuronal drive and lower information density in extrastriate visual areas, despite lower spiking noise, largely explain the impoverished visual sensitivity of primates near birth.

\section{Introduction}

Newborn primates have limited vision, and the maturation of their visual brain requires normal visual experience. However, many of the receptive-field (RF) properties of neurons in the primary visual cortex (V1) of infant monkeys are far more mature than previously thought (Blakemore and Vital-Durand,

Received Aug. 6, 2018; revised May 20, 2019; accepted May 22, 2019.

Author contributions: B.Z. and Y.M.C. designed research; Y.W., B.Z., X.T., G.S., and Y.M.C. performed research; Y.W., B.Z., X.T., and Y.M.C. analyzed data; Y.W., E.L.S., and Y.M.C. wrote the paper.

This work was supported by National Institutes of Health (National Eye Institute) Research Grants R01-EY-008128 (to Y.M.C.) and R01-EY-003611 (to E.L.S.), and Core Grant P30-EY-007551.

The authors declare no competing financial interests.

Correspondence should be addressed to Yuzo M. Chino at ychino@uh.edu.

https://doi.org/10.1523/JNEUROSCI.2023-18.2019

Copyright $@ 2019$ the authors
1981; Chino et al., 1997; Rust et al., 2002; Kiorpes et al., 2003; Kiorpes and Movshon, 2004; Zhang et al., 2005; Kiorpes 2015, 2016). As early as 2 weeks of age, V1 neurons are well tuned to stimulus orientation, spatial frequency, temporal frequency (TF), and binocular spatial phase disparity and by 4 weeks of age, qualitatively adult like tuning is present for most of these RF properties (Chino et al., 1997; Zheng et al., 2007; Maruko et al., 2008; Kiorpes, 2015). Also the center-surround receptive-field organization of individual V1 neurons is qualitatively adult like by $2-4$ weeks of age (Zhang et al., 2005).

Moreover, while the overall responsiveness (peak firing rate, dynamic range, and contrast sensitivity) of V1 neurons is quite impoverished, "information" carried by each action potential of V1 neurons is thought to be greater and more reliable than that by 
adult V1 neurons (Rust et al., 2002). More specifically, infant V1 neurons transmit information on stimulus contrast with higher fidelity than adult neurons because the variance-to-mean ratio of spiking is lower and information density (ID) is higher in infants (Rust et al., 2002). According to these authors, such robust reliability of spiking in infant V1 could potentially compensate for the lower firing rate and reduced contrast sensitivity of individual neurons. However, the behaviorally determined spatial contrast sensitivity of infant monkeys is only $\sim 20 \%$ of the adult level at $\sim 8-10$ weeks of age and remains quite poor (e.g., one-half of the adult level) at $\sim 30-40$ weeks of age (Kiorpes, 2016).

There are a number of neural factors that may limit the spatial contrast sensitivity of newborn infants other than reduced responsiveness of their V1 neurons. For example, immature responses of neurons beyond V1 are a distinct possibility and have been invoked to explain "poor" vision of young infants (Rust et al., 2002; Kiorpes and Movshon, 2004; Kiorpes, 2015, 2016). In adult monkeys, noisy spiking of extrastriate visual neurons is thought to limit information on sensory stimuli and potentially "hurt" perceptual performance (Shadlen and Newsome, 1998; Nawrot et al., 2008; Mitchell et al., 2009; Churchland et al., 2010; Kohn et al., 2016; Wang et al., 2017). Therefore, we explored the noise and the reliability of spiking in individual neurons of visual area V2, the earliest extrastriate visual area, to understand the neural basis of the poor contrast sensitivity of young infants. Specifically, we analyzed the spiking patterns of individual V2 neurons by quantifying spiking dynamics, contrast sensitivity, spiking noise [irregularity of interspike intervals (ISIs) and trialto-trial fluctuations], and the amount of information on stimulus contrast carried by each spiking (information density).

\section{Materials and Methods}

Procedures. All experimental and animal care procedures were in compliance with the Guiding Principles for Research Involving Animals and were approved by the Institutional Animal Care and Use Committee of the University of Houston.

Subjects. The subjects were rhesus monkeys (Macaca mulatta): two infants ( 4 and 8 weeks old, both female) and three adult monkeys (one male and two females). Much of the data for adult monkeys were obtained during previous projects (Tao et al., 2012, 2014; Zhang et al., 2013; Wang et al., 2017).

Recording and stimulation. The surgical preparation and the recording and stimulation methods were described previously in detail (Maruko et al., 2008; Tao et al., 2012, 2014; Zhang et al., 2013; Wang et al., 2017). Briefly, monkeys were anesthetized initially with an intramuscular injection of ketamine hydrochloride (15-20 mg/kg) and acepromazine maleate $(0.15-0.2 \mathrm{mg} / \mathrm{kg})$. The animals were paralyzed by an intravenous infusion of vecuronium bromide (a loading dose of $0.1-0.2 \mathrm{mg} / \mathrm{kg}$ followed by a continuous infusion of $0.1-0.2 \mathrm{mg} / \mathrm{kg} / \mathrm{h}$ ) and artificially respired with a mixture of $59 \% \mathrm{~N}_{2} \mathrm{O}, 39 \% \mathrm{O}_{2}$, and $2 \% \mathrm{CO}_{2}$. Anesthesia was maintained by the continuous infusion of a mixture of sufentanyl citrate $(0.05 \mu \mathrm{g} / \mathrm{kg} / \mathrm{h})$ and propofol $(4 \mathrm{mg} / \mathrm{kg} / \mathrm{h})$. The core body temperature was kept at $37.6^{\circ} \mathrm{C}$. Cycloplegia was produced by $1 \%$ atropine sulfate, and the corneas of the animals were protected with rigid, gas-permeable, extended-wear contact lenses. Retinoscopy was used to determine the contact lens parameters required to focus the eyes on the stimulus screen.

A monitor (Vision Research Graphics) with ultrashort persistence (frame rate $=140 \mathrm{~Hz}, 800 \times 600$ pixels) was used to present visual stimuli. The viewing distance was set to $114 \mathrm{~cm}$, where the display subtended a visual angle of $20^{\circ}$ (horizontal) $\times 15^{\circ}$ (vertical). The mean luminance of the monitor screen was kept at $60 \mathrm{~cd} / \mathrm{m}^{2}$. Multiunit or single-unit activities were extracellularly recorded with tungsten-in-glass microelectrodes (FHC). Action potentials from individual cortical neurons were amplified, digitized at $25 \mathrm{kHz}$, and stored using the data acquisition components in our workstation (System 3, Tucker-Davis Technology). During recording experiments, the spike-sorting software in our data acquisition system (System 3, Tucker-Davis Technology) was used to maintain the quality of isolation and to ensure that we were recording from the same units throughout each run. After the completion of the entire experiment for a given monkey, custom-made spikesorting software was used off-line to better isolate spiking activity.

Experiments. Neuronal responses to drifting sine-wave gratings were sampled at a rate of $140 \mathrm{~Hz}$ (7.14 ms bin widths) and compiled into peristimulus time histograms (PSTHs) that were equal in duration to, and synchronized with, the temporal cycle of the grating. The amplitude and the phase of the temporal response components in the PSTHs were determined by Fourier analysis. For each neuron, the orientation, spatial frequency, and size-tuning functions were first obtained using sine-wave gratings ( $80 \%$ contrast; temporal frequency, $3.1 \mathrm{~Hz}$ ). The preferred orientation, spatial frequency, and size for each receptive field were determined using the methods described previously in detail (Tao et al., 2012, 2014). For the noise measurements, the optimized stimuli for each neuron were presented to the right or left eyes in a randomly ordered sequence for $640 \mathrm{~ms}$. Rerandomized stimulus sequences were repeated 25 times during a given experiment. Blank stimuli were included in each repeat to provide a measure of the maintained firing rate (spontaneous firing) of a neuron. We repeated the measurements for the following stimulus contrasts: $0.0 \%, 1.0 \%, 2.5 \%, 5 \%, 10 \%, 25 \%, 50 \%$, and $80 \%$.

Spiking dynamics. The analysis includes the following: (1) the baseline firing (base); (2) the onset latency; (3) the time to reach one-half of the peak firing (time to half-peak); (4) the time to reach the peak firing (time to peak); and (5) the decay in firing (decay), defined as the ratio between the responses at peak and platform and calculated as decay $=($ peak platform $) /($ peak + platform $-2 \times$ base; see Fig. $2 A)$. The onset latency was determined by measuring the time between stimulus onset and the time at which the response of the neuron significantly exceeded the background noise distribution: specifically, over three consecutive $1 \mathrm{~ms}$ bins, exceeding a Poisson distribution of spontaneous spiking measured for a period of $100 \mathrm{~ms}$ preceding the stimulus onset and at a level corresponding to a probability of $p=0.01$ (Maunsell and Gibson, 1992).

For better resolution, spike count data were presented as impulse density functions produced by smoothing the $1 \mathrm{~ms}$ PSTHs with a discretetime Gaussian pulse with an SD of 3 ms. The spiking histograms represent the spike counts for all cells and all 25 repeats. The same number of cells from each group for infants and adults were randomly resampled, and their latency, time to half peak, and time to peak were calculated 2000 times. The median estimated timing and their confidence intervals were calculated from these 2000 repeats. A bootstrap method was used to test for significant differences between cell groups. This was performed by mixing random cells from the two cell groups as the total number of neurons for the smaller group and the timings for the new sampling population were calculated. This procedure was performed 20,000 times, and the results sorted. The original timing ranked $0.5 \%$ or $99.5 \%$ of the repeat pool and was identified as significantly different from the other group.

Contrast versus response. With optimized drifting gratings, we plotted the response amplitude of each unit as a function of stimulus contrast. We fit contrast response data with the following hyperbolic function:

$$
G(C)=R_{\max } C^{n} /\left(C_{50}^{n}+C^{n}\right)+R_{\mathrm{sp}}
$$

where $R_{\max }$ is the maximum attainable response, $C_{50}$ is the semisaturation contrast or a relative position of the function on contrast $(x)$ axis, and $n$ is the slope of the function. We constrained our fit by including "zero data points" [i.e., forcing data points that are 2 SEs below the noise (mean spontaneous activity) to zero]. The contrast threshold was operationally defined as the contrast at which the fit function intersected the response level of 2 SEs above the noise (see Fig. 3A). There were substantial numbers of V1 and V2 neurons in infants that did not have spontaneous activity. For such units, contrast threshold ("hard threshold") was defined as the contrast at which the fit function intersected " 0 " discharge.

Noise in spiking. Spiking noise was assessed by measuring the spike count variability [the square of the coefficient of variation $\left(\mathrm{CV}^{2}\right)$ ] and trial-to-trial variability [Fano factor (FF)]. We quantified the spike count variability by calculating the square of the coefficient of variation of the 
ISIs within the $640 \mathrm{~ms}$ stimulus window. Importantly, the $\mathrm{CV}^{2}$ of operational ISIs quantifies spike train irregularities within trials. Operational spike timing was obtained by using the cumulative smoothed peristimulation histogram $(\lambda)$ for each cell at original timing as follows: $t^{\prime}$

$\int_{0}^{t} \lambda(s) d s$. The operational ISIs represent the intervals between their operational timing $\left(\Delta t^{\prime}\right)$. ISIs were sequenced for all trials within a $640 \mathrm{~ms}$ stimulation window. The variance and mean of the operational ISI sequence were then calculated to obtain $\mathrm{CV}^{2}=\operatorname{Var}\left(\Delta t^{\prime}\right) / E\left(\Delta t^{\prime}\right)^{2}$ for each cell (Nawrot et al., 2008). The FF of the spike count reveals spiking irregularity between trials (trial-to-trial fluctuations), and meanmatched Fano factor (m-FFs) were calculated (Churchland et al., 2010). The m-FFs were processed from the histograms of mean spike counts for all cells within a time window of interest for each cell group. At each spike-count bin, the same number of cells as the smallest group was subsampled. Combining cells from all bins, the FF was calculated as the least-squares solution for $\operatorname{Var}_{i}=\mathrm{FF} \times E_{i}$. The average FFs and average estimated SEs of 50 resamplings were defined as $\mathrm{m}-\mathrm{FF}$ and its MSE. The $p$ values between $\mathrm{m}$-FF groups were computed as the normal cumulative distribution function of the following:

$$
\sigma=\sqrt{\mathrm{MSE}_{1}^{2}+\mathrm{MSE}_{2}^{2}} \text { at }-\left|\mathrm{mFF}_{1}-\mathrm{mFF}_{2}\right| .
$$

Sliding windows of 50 and $200 \mathrm{~ms}$ at $10 \mathrm{~ms}$ steps were used to measure the spike counts and FFs to characterize the dynamics of spiking rate and spiking noise before, during, and after stimulation. Because the shorter sampling window includes fewer spikes compared with the full $640 \mathrm{~ms}$ stimulation period in some cases, and there were no spikes across all trials within our 25 repeats, those cells and time bins would not have valid $\mathrm{CV}^{2}$ and FF values. As an impact for the group total, both $\mathrm{CV}^{2}$ and FF would be underestimated compared with real values. Therefore, the data from those cells were not included in the analysis.

Mutual information and information density. We calculated the mutual information and information density by applying a similar approach to that developed by Rust et al. (2002). We quantified the mutual information about contrast by calculating the difference between the total entropy across all contrasts and the mean noise entropy at each contrast by applying the following equation:

$$
I=-\sum_{r} P(r) \log _{2} P(r)+\sum_{s} P(r \mid s) \log _{2} P(r \mid s),
$$

where $r$ is the number of spikes in a $640 \mathrm{~ms}$ trial and $s$ is the contrast level of gratings. This equation was used to calculate pairwise information, approximately two selected contrasts including $0 \%$ (spontaneous), $5 \%$, $10 \%, 25 \%, 50 \%$, and $80 \%$. To quantify information density, we calculated mutual information for all possible pairs of contrasts (six contrasts, 15 pairs) from spike counts in $640 \mathrm{~ms}$ bins. We fit the relationship between mutual information and the difference in spike count with the following function:

$$
I=\left[1-(1-\alpha)^{\Delta n \beta}\right] \log _{2} S,
$$

where $I$ is the mutual information, $\Delta n$ is the difference in spike count, $S$ is the number of stimuli (two), and $\alpha$ and $\beta$ are free parameters. The maximum slope of this function represents the peak rate of information growth with difference in spike count, and this quantity is defined as ID (Rust et al., 2002). We used the least-squares fit to estimate optimized fitting for Equation 2, then searched for the point with maximum slope in this curve, which is defined as information density.

Statistics. For the cell group comparison between infants and adults, we used Student's $t$ test or one-way ANOVA and post hoc Bonferroni tests if results were based on the normally distributed parametric data with equal variance between the cell groups. Descriptive statistics for these analyses are provided as the mean \pm SEM. For the data that did not meet the criteria, a Mann-Whitney rank sum test or Kruskal-Wallis one-way ANOVA on ranks was used. This initial test was followed by a Dunn's post hoc test, with data presented as the median \pm interquartile range. For the complex character of population data, such as mean-matched Fano factor and global information density, we used reshuffling methods. For this analysis, we randomly resampled data 2000 times from subgroup data and calculated their population characters. The mean value was taken as the population mean, and the standard variation was taken as the SEM. Then we applied a two-sample $z$ test to check for significance. For the analysis of data on response timing, if the population character did not meet the criteria for normal distribution, we used bootstrap methods as described above.

\section{Results}

We recorded from 178 V2 neurons in two infant monkeys and 211 units in three normal adult monkeys. By using our highly efficient on-line and off-line spike sorting methods (Zhang et al., 2013; Tao et al., 2014; Wang et al., 2017), similar to the approach described by Martin and Schröder (2013), we quantitatively analyzed the responses of a well isolated single unit. The data from two infant monkeys (55 neurons from 4-week-old infants and 123 neurons from 8 -week-old infants) were combined since there was no significant difference in any of the response measures described in this study. We analyzed the responses to stimulations via the dominant eye. Electrode penetrations were confined to a parasagittal plane, and the angle of the penetration was typically $15^{\circ}$ from vertical. The penetrations were started right behind the blood vessels running along the lunate sulcus, and ended when the electrode exited V2. For each penetration, the cortical depth for each isolated unit was recorded. This approach allowed us to sample units consistently from similar regions of V2 in infants and adults. Nearly all receptive fields were located within $5^{\circ}$ of the center of the projected fovea for both infants and adults. To analyze the dynamics of spike counts and spiking noise, we presented brief $(640 \mathrm{~ms})$ drifting $(\mathrm{TF}=3.1 \mathrm{~Hz})$ sine-wave gratings optimized for orientation, spatial frequency, and size for each neuron. We varied stimulus contrast between $0 \%$ and $80 \%$; each contrast was presented 25 times.

\section{Dynamics of spike counts}

The spiking dynamics of infant neurons exhibit considerable immaturity, especially the ability of a cell to efficiently integrate input signals. The spike counts (mean $\pm \mathrm{SE}$ ) for a range of stimulus contrasts revealed substantial differences between infants and adults (Fig. 1). In addition to the overall firing rate, the most notable difference was that the onset transient discharge was absent in infants even for the highest stimulus contrast (80\%; Fig. $1 B$ ). In adult monkeys, the transient responses are clearly visible for all contrasts except for weak transient responses for $10 \%$ contrast (Fig. 1A).

Also the onset latency and the time to reach the one-half of the peak response amplitude were much longer for infants than for adults. Moreover, unlike the relatively rapid decline of response rates after the peak in adults, the response decay in infants occurred only after the stimulus was turned off. The running $t$ values provide a clearer picture of the differences in the dynamics of spike counts between the cell groups (Fig. 1C). The largest difference between infants and adults was located around the peak response (Fig. 1C, filled arrow), whereas minimal differences occurred around stimulus onset and at $\sim 160 \mathrm{~ms}$ after stimulus offset when responses became minimal for both infants and adults (Fig. $1 C$, small open arrows).

The above differences in spike count dynamics between infants and adults are well reflected in the cell population data (Fig. 2 ). The onset latency of infant neurons was much longer than that in adults for all contrasts $(p<0.001$; Fig. $2 B)$. Similar prolonged latencies (longer integration time) were previously reported in infant V1 (Rust et al., 2002). The longer onset latency in infants was especially notable for lower stimulus contrasts $(10 \%$ and 
$20 \%$ ). Similar differences between infants and adults were found for the time to reach the half peak (Fig. 2C), except for $10 \%$ contrasts $(p<0.001)$. Also, the average time to reach the peak response (time to peak) for infant neurons was significantly longer than that for adult neurons for all contrasts $(p<$ 0.01; Fig. 2D).

Importantly, the response decays after the peak, measured by the peak/platform amplitude ratios (Fig. 2A), were also lower (i.e., slower decay) in infant neurons for higher contrast gratings $(50 \%$ and $80 \%$; $p<0.01$; Fig. $2 E$ ), whereas the decay was longer in infants at $10 \%$ and no statistical difference was found for $25 \%$ contrast. It is important to note that some of the apparently "inconsistent" data for $10 \%$ contrast were likely to be due to the very low firing rates of infant neurons (Figs. 3C, $4 C)$. The results for high-contrast stimuli (50\% and 80\%) largely reflect the aforementioned lack of onset transient discharges in infant neurons.

\section{Contrast versus response}

To reveal the unique nature of the immaturity in the contrast sensitivity of V2 neurons, we fit the contrast versus response data for each neuron with a hyperbolic function and calculated the contrast threshold, the semisaturation constant, or overall contrast sensitivity $\left(C_{50}\right)$, the contrast gain, or the slope of the contrast response function $(n)$, and the saturated response or maximum attainable response $\left(R_{\max }\right.$; Fig. $\left.3 A\right)$. Example neurons from infants in Figure $4 B$ show that their contrast thresholds were higher and $C_{50}$ positions were shifted toward higher contrasts, but $R_{\max }$ values were lower compared with adult neurons. Not surprisingly, the average response rate for infant neurons was consistently lower for all stimulus contrasts than that for adult neurons ( $p<0.001$; Fig. $3 C$ ). The spontaneous firing rate $(0 \%)$ was nearly identical in infants and adults. Interestingly, the average response rate in adults saturated beginning at $\sim 25 \%$ contrast, whereas infant neurons showed exceedingly small saturation even for $80 \%$ contrast (for more details, see below). However, the dynamic range (the range between the lowest and highest response rates) was greater for adults than for infants (see Fig. $7 F$ ).

The cell population analysis shows that median contrast threshold of infant neurons was nearly five times higher than that of adult neurons (Fig. 3D; Wilcoxon rank sum test, $p<0.001$ ). Similarly, the overall contrast sensitivity was lower $\left(C_{50}\right.$ was higher) for infants (Wilcoxon rank sum test, $p<0.001$ ) in part because a substantial number of infant V2 neurons (29\%) had $C_{50}$ values of $>100 \%$ (Fig. $3 E$ ).

There was a significant difference in median $R_{\max }$ between infants and adults (Wilcoxon rank sum test, $p=0.032$; Fig. $3 F$ ) due to the large variability in the infant cell population (for more details, see below). The contrast gains $(n)$ were slightly lower for infants, but there was no statistically significant difference between infants and adults (Wilcoxon rank sum test, $p>0.05$; Fig. 3G).

Unlike adult V2 neurons, many infant V2 units (20\%) did not show response saturation for high-contrast gratings and as a result, their $R_{\max }$ values exceeded 100 spikes/s (Fig. $3 F$ ). It is important to keep in mind that $R_{\max }$ signifies the maximum attainable response rate of a cell obtained from its contrast versus response function, but does not necessarily signify the actual peak response of the cell (Fig. $4 C$ ). The contrast versus response functions of three example neurons from infants are illustrated in Figure $4 A$ to make this point. Compare the $R_{\max }$ value of each cell with its firing rate measured at $80 \%$ contrast. The frequency distribution of the maximum firing rates for infants and adults (Fig. $4 C$ ) clearly shows that the mean firing rate of infant units was significantly lower than that for adults $(p<0.001)$. Finally, a large proportion of infant units $(31 \%)$ had $R_{\max }$ values that were $<1.0$ spike/s (Fig. $3 F$ ). Figure $4 B$ illustrates the contrast versus response functions of two representative neurons. These units were not responsive to low-to-middle contrast stimuli, and their responses saturated rapidly for high-contrast stimuli.

Since a substantial proportion of adult V2 neurons $(\sim 10 \%)$ had rather low firing rates (Fig. 4C) and small dynamic ranges 

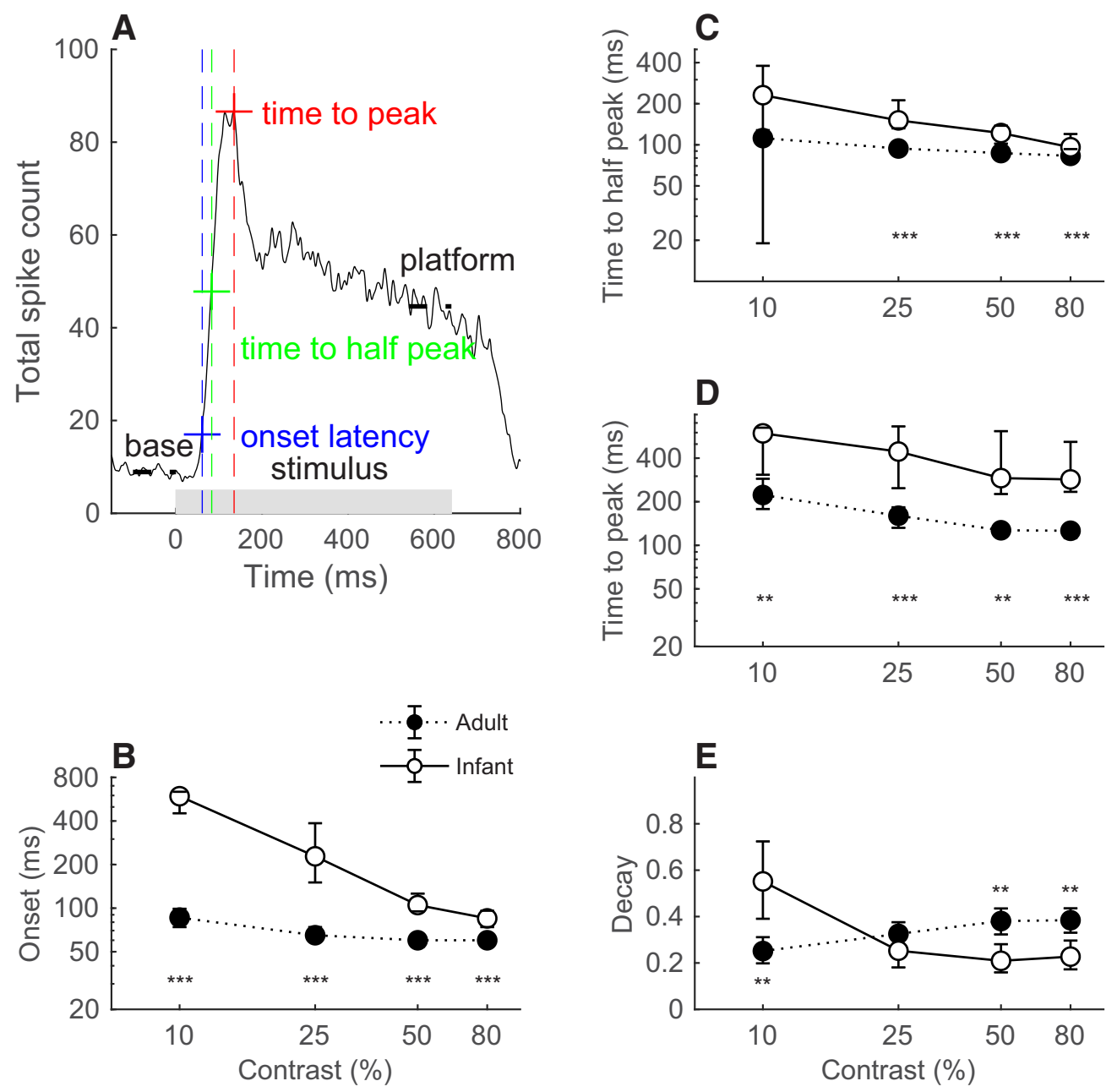

Figure 2. A, Methods to measure dynamics of spike counts. Example cell onset latency (blue vertical dashed line), time to half-peak (green vertical dashed line), time to peak (red vertical dashed lines), and spontaneous "base" response and "platform" (thick horizontal dashed lines). Thick gray bar at the bottom indicates stimulation duration. Horizontal thin lines indicate thresholds for each measure. $\boldsymbol{B}-\boldsymbol{E}$, Cell population data on onset latency $(\boldsymbol{B})$, time to half-peak $(\boldsymbol{C})$, time to peak $(\boldsymbol{D})$, and decay $(\boldsymbol{E})$. Error bars show confidence intervals $\left({ }^{* * *} p<0.001,{ }^{* *} p<0.01\right)$.

(see Fig. 7F), it is important to state here that the responses of these adult neurons (e.g., dynamic ranges $<2.0$ spikes/s) were stimulus driven and significant (ANOVA) and that the goodness of fit $\left(R^{2}\right)$ for these neurons was also significant.

\section{Irregularities in spike train $\left(\mathrm{CV}^{2}\right)$}

Spiking noise reflects irregularities in the spike train and trial-totrial variability (Nawrot et al., 2008; Churchland et al., 2010; Wang et al., 2017). Here we quantified the spiking irregularity $\left(\mathrm{CV}^{2}\right)$ by calculating the square of the coefficient of variation of the ISIs within the $640 \mathrm{~ms}$ stimulus window. Specifically, we calculated the operational time $\mathrm{CV}^{2}$ (for details, see Materials and Methods). This analysis method minimizes the effects of temporal changes in firing due to adaptation and/or stimulation that could result in overestimation of $\mathrm{CV}^{2}$ ("demodulation"; Nawrot et al., 2008).

The $\mathrm{CV}^{2}$ was significantly lower for $\mathrm{V} 2$ neurons in infants than that in adults for all stimulus contrasts $(p<0.001$; Fig. $5 A)$. We found much lower variance in the operational ISI $(p<0.001$; Fig. $5 B)$ and also the lower square mean of ISI $(p<0.001)$ for all stimulus contrasts in infants (Fig. $5 C$ ). However, the difference between infants and adults in the degree of spike train irregularity $\left(\mathrm{CV}^{2}\right)$ mainly resulted from relatively grater differences in the variance of the operational ISI (Fig. 5B). Also, the differences in
$\mathrm{CV}^{2}$ between infants and adults were contrast dependent; the largest difference was found not for $80 \%$ contrast, but for $50 \%$, and the smallest difference was found for the highest contrast $(80 \%)$. This is because $\mathrm{CV}^{2}, \mathrm{VAR}_{\mathrm{ISI}}$, and $\mathrm{E}_{\text {ISI }}^{2}$ all peaked at $50 \%$ contrast for adults, whereas in infants all these values were higher for $80 \%$ contrast.

Interestingly, the $\mathrm{CV}^{2}$ during spontaneous firing (contrast $=$ $0.0 \%)$ for infants was much lower than that for adults $(p<0.001$; Fig. $5 A$ ). This difference occurred largely because the square mean of ISI was significantly greater for infants compared with that for adults $(p<0.001$; Fig. $5 C)$, while the variance of ISI in infants was slightly lower in infants (Fig. $6 B$ ).

\section{Trial-to-trial variability}

To assess the trial-to-trial variability of spiking, we calculated the variance-to-mean ratios, or Fano factor, of infant V2 neurons using a rate-matching approach (Churchland et al., 2010). Stimuli were $640 \mathrm{~ms}$ in duration and repeated 25 times with a rest period of $320 \mathrm{~ms}$. Temporal changes in m-FF were quantified using spike counts computed in a $200 \mathrm{~ms}$ sliding window moving in $10 \mathrm{~ms}$ steps. Data are aligned on stimulus onset (for more details, see Materials and Methods).

Consistent with a previous report on infant V1 (Rust et al., 2002), the variance-to-mean ratio of V2 neurons for our infant 
A

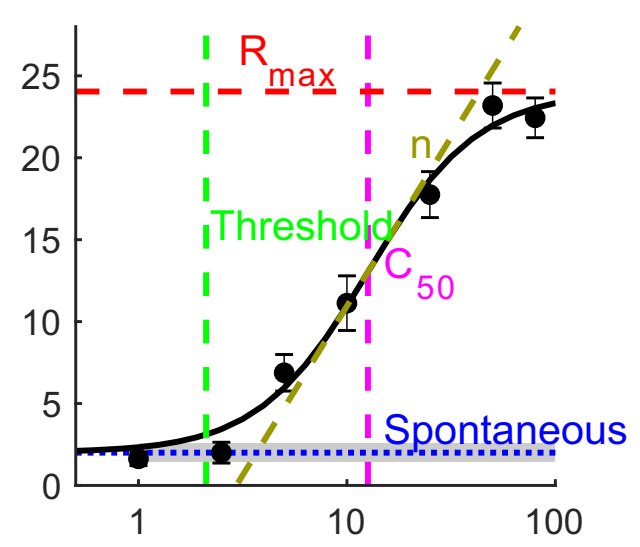

B
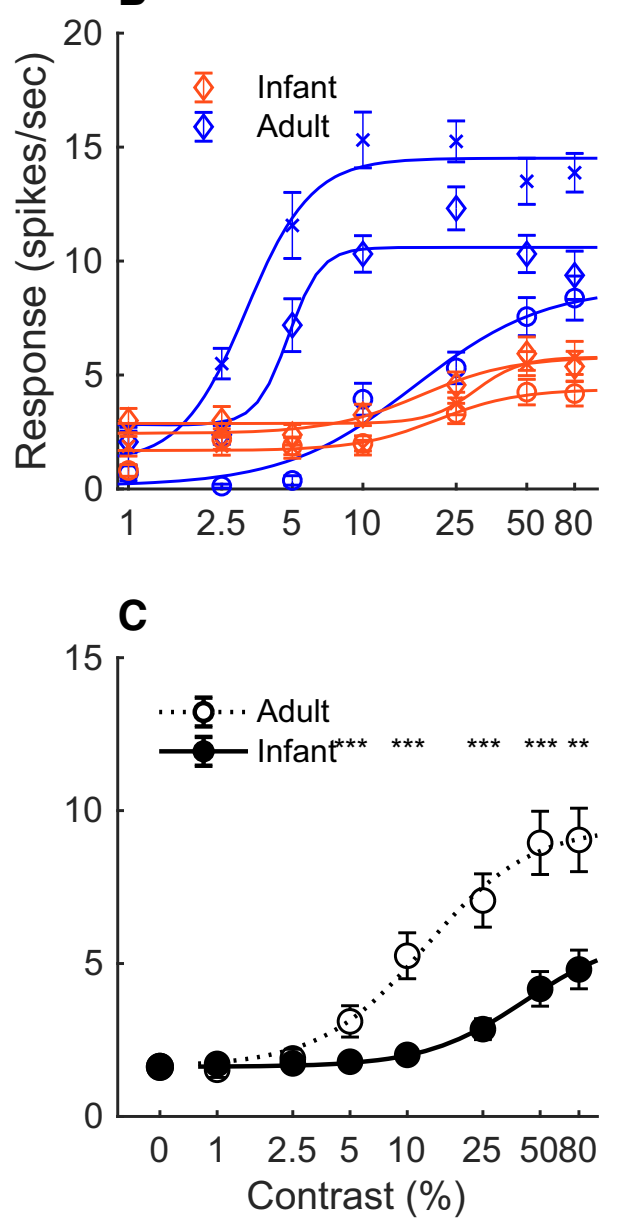

D
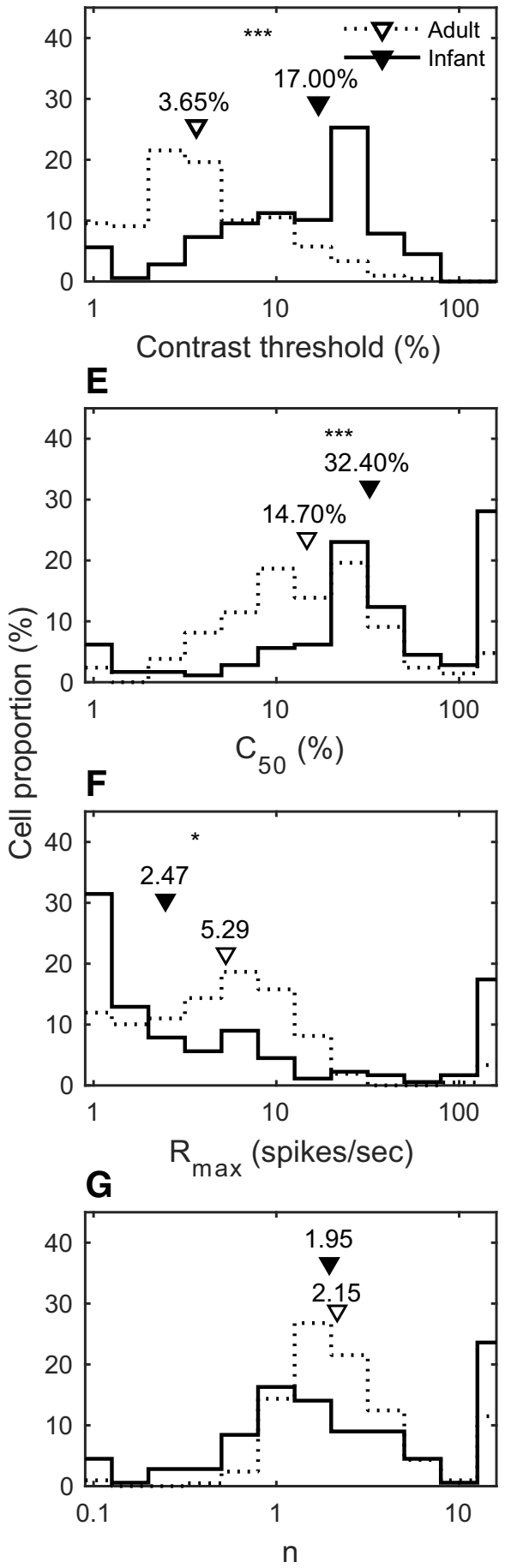

Figure 3. Contrast sensitivity. $\boldsymbol{A}$, Contrast vs response function of an example cell. Horizontal dotted line indicates spontaneous response, and gray shading shows confidence interval. Vertical green dashed line shows contrast threshold. Horizontal red dashed line indicates the estimated $R_{\max }$. Vertical magenta dashed line shows $C_{50}$. $B$, Contrast vs response functions of example neurons for infants (red) and adults (blue). C, Mean ( $\pm \mathrm{SE}$ ) response rates of all adult and infant cells for different contrasts $\left({ }^{* * *} p<0.001,{ }^{* *} p<0.01,{ }^{*} p<0.05\right.$ ). D, Distributions of contrast threshold. $E, C_{50} \cdot \boldsymbol{F}, R_{\max } \cdot G$, Gain index, $n$. Downward triangles indicate the median values of each cell group.

monkeys was much lower than that for adult monkeys (Fig. 5D). However, the important difference between the two studies is that, unlike in the previous study in V1 (Rust et al., 2002) or V2 (Zhang et al., 2008), we used the mean-matched Fano factor analysis (Churchland et al., 2010), and therefore, the large differences in mean firing rates between infants and adults in this study (Fig. 4C) had little or no effect on the analysis of trial-to-trial variability of spiking.
The cell population data revealed additional key differences between infants and adults (Fig. 5D-F). The average m-FF for infants was significantly lower than that for adults for all stimulus contrasts $(p<0.01)$. Moreover, the largest difference was observed for the lowest stimulus contrast (10\%). During spontaneous firing $(0 \%)$, the difference in average $\mathrm{m}$-FF between infants and adults was even greater than that for $10 \%$ contrast (Fig. $5 D$ ). Also both the variance of spike counts and the mean spike counts 

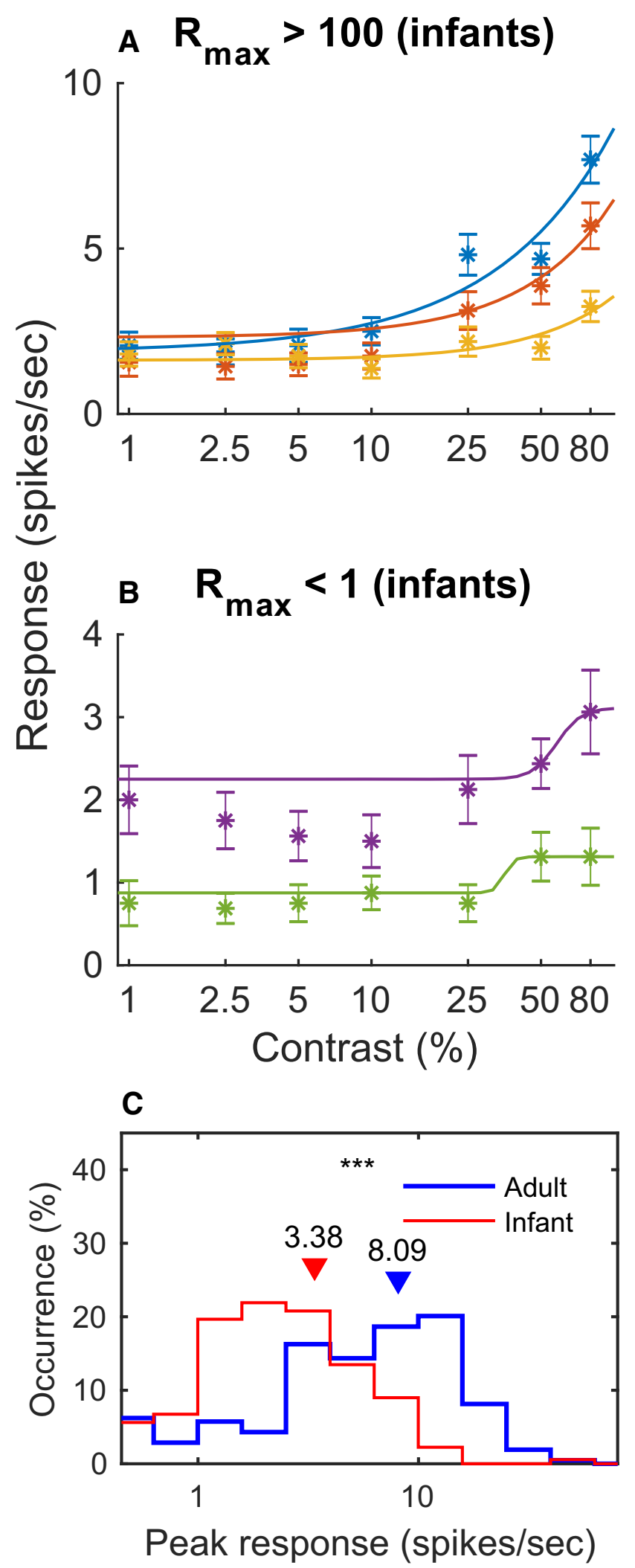

Figure 4. $R_{\max }$ vs peak response rate. $A, B$, Contrast vs response functions of example neurons from infants that had $R_{\max }$ of $>100$ spikes $/ \mathrm{s}(\boldsymbol{A})$, and $R_{\max }$ of $<1.0$ spikes $/ \mathrm{s}(\boldsymbol{B})$. Note that none of the units with $R_{\max }$ values $>100$ spikes had recorded a firing rate of $>10$ spikes $/ \mathrm{s}$. $\boldsymbol{C}$, Distribution of peak response rate of all neurons from adults (blue) and infants (red). Triangles indicate means. ${ }^{* * *} p<0.001$. were significantly lower in infants than in adults $(p<0.001$; Fig. $5 E, F)$. The relatively larger difference in variance of spike counts between infants and adults, compared with the similar difference in mean spike counts, resulted in the observed reduced average $\mathrm{m}$-FF for infants. The elevated $\mathrm{m}$-FF during spontaneous firing in adult $\mathrm{V} 2$ neurons can be attributed to a higher variance of spike counts in adult V2 neurons compared with that in infants $(0 \%$; Fig. 5E).

\section{Information density}

Another way to assess the reliability of spiking signals is to quantify information density, the amount of information conveyed by each action potential on contrast (Rust et al., 2002). More specifically, we determined whether the information density for our V2 neurons was higher in infants than in adults, as previously reported for V1 (Rust et al., 2002). To facilitate comparisons with the previous study in $\mathrm{V} 1$, we chose six stimulus contrasts $(0 \%$, $5 \%, 10 \%, 25 \%, 50 \%$, and $80 \%$ ) and calculated mutual information for each neuron as a function of spike count differences for 15 pairs of comparisons.

\section{Global information density}

Because V2 neurons exhibit different dynamic ranges for infants and adults, and the ID of V2 neurons regardless of age does not follow normal distribution ( $p<0.001$, Jarque-Bera test), we used a "mean matched method" to compare the information density for cell populations (Fig. 6A). With this approach, both cell groups have identical distributions of response differences (Fig. $6 \mathrm{~A}$, sample size). For both cell groups, we created histograms for all spike rate differences for 20 connected bins. Each bin is expected to have an equal number of total occurrences. A varied number of identical spike rate differences fell on the border of bins, and, as a result, the total number of counts (occurrence) was not equal across bins. The first bin includes all 0 spike rate difference, which was much larger than all other bins but counts were approximately equal for infants and adults. The last bin includes the maximal spike count differences, which were far greater for adults than for infants. To avoid unequal sampling, we subsampled the same number of points from each bin using the total number of counts for the smaller group. Least-squares fitting of Equation 2 in Materials and Methods was repeated 200 times using different random resampling, and the mean matched information density ("global information density") was obtained for adults and infants.

The scatter plot in Figure $6 B$ relates the spike count difference with mutual information of each cell. All data points were fit with a mean-matched function for infants and adults (solid curves), and the straight thick dash line shows the slope of the steepest portion of the fit function for each cell group (i.e., global information density). The global information density was significantly lower for infants $(0.1219)$ than for adults $(0.1409 ; z$ test for mean matched fitting, $\left.p<1.154 \times 10^{-30}\right)$.

Since this result in V2 was inconsistent with the previous observation reported for V1 by Rust et al. (2002), we calculated information density for each cell as was done in their previous study. To quantify information density, we calculated mutual information for all possible pairs of contrasts (six contrasts, 15 pairs) from spike counts in $640 \mathrm{~ms}$ bins. We fit the relationship between mutual information and the difference in spike count by using the least-squares fit to estimate optimized fitting for Equation 2, as described above. Then we searched for the point with maximum slope in this curve, which is defined as ID (Rust et al., 2002; for more details, see Materials and Methods). 


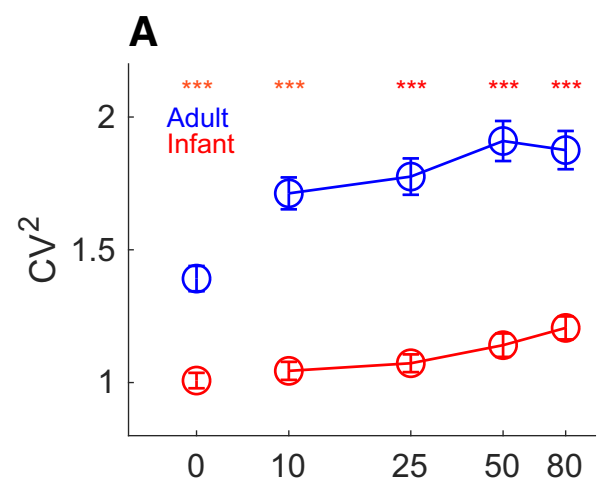

B

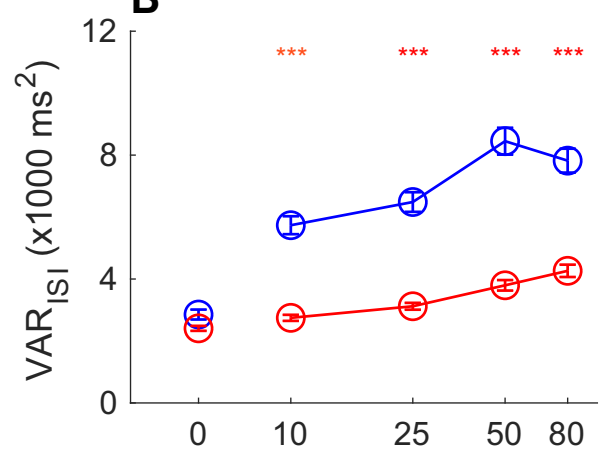

C

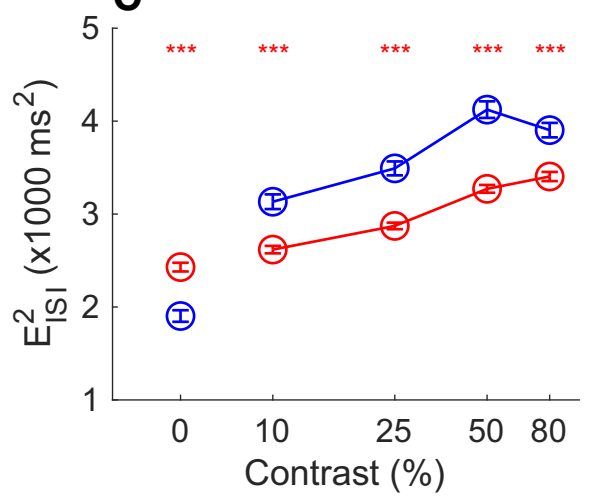

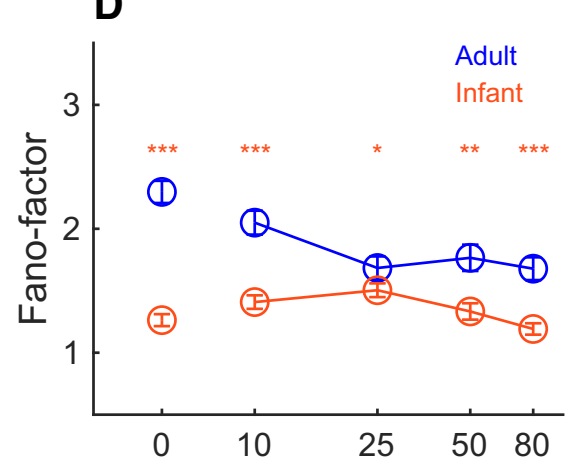

E
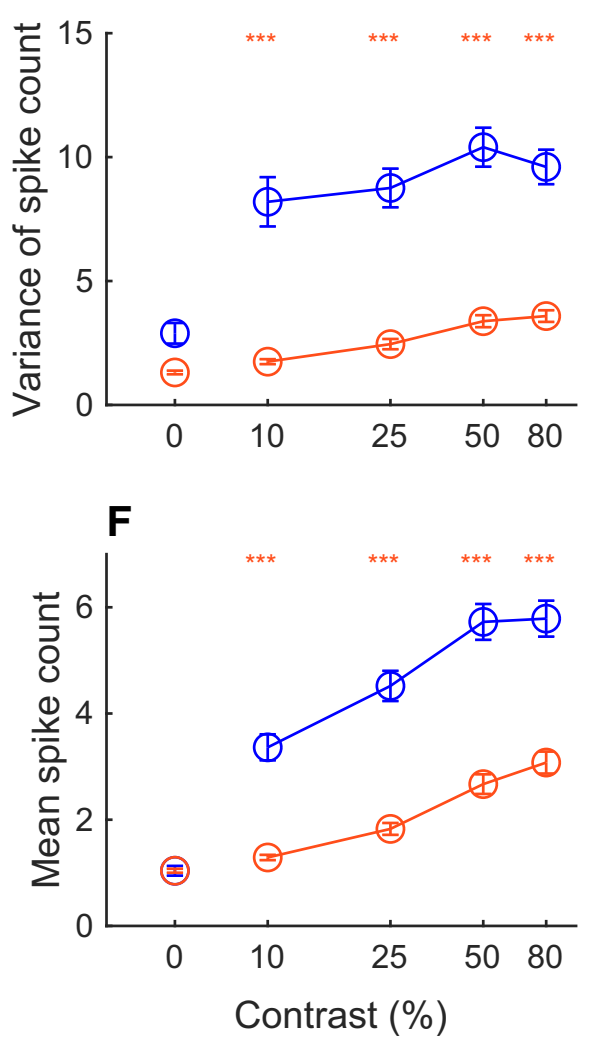

Figure 5. Operational $C V^{2}$ and Fano factor as a function of contrast. $A$, Operational $\mathrm{CV}^{2}$. B, Variance of operational ISI. C, Squared mean of operational ISI. D, Population average of Fano factors. $E$, Variance of spike count. $F$, Mean of spike count. Error bars show $S E\left({ }^{* * *} p<0.001,{ }^{* *} p<0.01,{ }^{*} p<0.05\right)$. Centers of circles indicate mean of each cell groups.

Figure $6 \mathrm{~A}$ illustrates the mutual information as a function of differences in spike count between pairs of contrasts for those six neurons that are shown in Figure $3 B$ (contrast vs response functions). All three adult neurons had higher information density than did infant neurons. Similar functions are illustrated for five infant neurons that exhibited "exceptional" $R_{\max }$ values, specifically $R_{\max }>100$ spikes/s and $<1.0$ spikes/s [compare Figs. $4 A, B$, $7 B$ (by using color)]. The information densities for these exceptional infant neurons were similar to those neurons exhibiting "typical" contrast versus response functions (Fig. 7A).

Consistent with the data for the example neurons (Figs. $7 A, B$ ) and the global fitting (Fig. 6), the average information density of individual V2 neurons for infants $(0.1407 \pm 0.0045)$ was significantly lower than that for adults $(0.1521 \pm 0.0076$; $p<0.05$; Fig. $7 C$ ). However, in $\mathrm{V} 1$, the average information density is $\sim 0.2$ for infants of comparable ages, whereas for adults it is $\sim 0.1$ (Rust et al., 2002). While the average information density of V2 neurons was higher for adults than for infants in this study, the difference in the average information density in V2 was much smaller than that in V1.

Next, we determined how the information density of individual V2 neurons is related to irregularities in their spike train $\left(\mathrm{CV}^{2}\right)$ and trial-to-trial variability $(\mathrm{FF})$. The scatterplot in Figure $7 D$ shows the relationship between information density and irregularities in the spike train $\left(\mathrm{CV}^{2}\right)$ for all units. For adults, there was a relatively strong inverse correlation between information density and $\mathrm{CV}^{2}(r=-0.47, p<0.0001)$. However, this relationship was substantially weaker for infants $(r=-0.21, p=0.0043)$, in part because many infant units with lower spiking noise $\left(\mathrm{CV}^{2}\right)$ did not have higher information density.

Not surprisingly, the information density of individual V2 neurons was also inversely correlated with their variance-tomean ratios (mean matched Fano factor) in both infants and adults (Fig. 7E). This correlation was similar for both groups but 
A

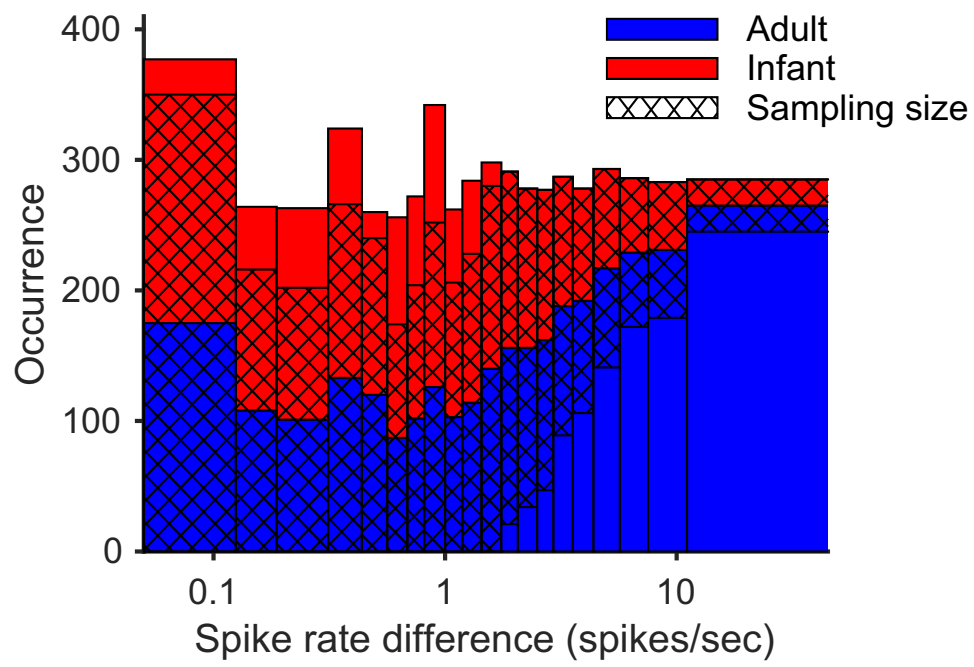

B

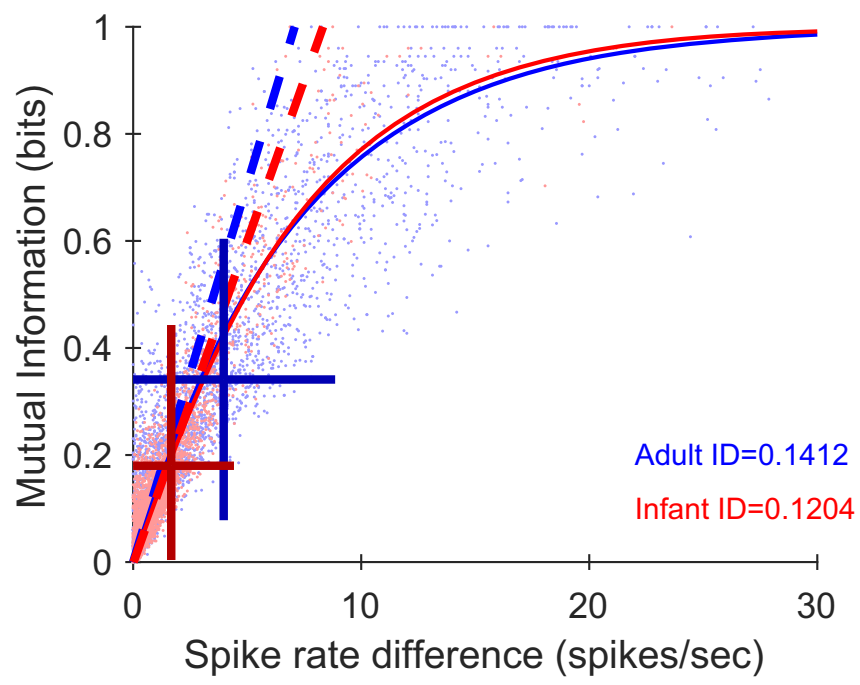

\section{Discussion}

Dynamics of spike counts

The lack of transient discharge for drifting gratings in infants (Fig. 1) may reflect the immaturity of the microcircuitry of V2 neurons. The receptive field of an adult $\mathrm{V} 2$ neuron is made up of many subunits that reflect feedforward V1 input (Ito and Komatsu, 2004; Anzai et al., 2007; Zhang et al., 2013). Also, V2 neurons receive signals from neighboring units via the intrinsic long-range connections and from higher-order visual areas (Baldwin et al., 2012; Zhang et al., 2014). The intrinsic long-range connections in V2 of young infants are immature (Zhang et al., 2005; Baldwin et al., 2012), and higher-order visual areas are relatively underdeveloped soon after birth (Kiorpes and Movshon, 2004; Zhang et al., 2005). Together, the "integration" of numerous input signals at stimulus onset could be less precise and gradual, hence, leading to slower onset latencies and the absence of onset transient responses (Figs. 1, 2). Also the neural mechanisms that initiate the rapid decline in spiking rate from the peak firing might be immature; e.g., lower synaptic depression (Müller et al., 2001; Carandini et al., 2002; Boudreau and Ferster, 2005), underdeveloped intrinsic membrane properties that are responsible for contrast adaptation (Carandini and Ferster, 1997, 2000; Sanchez-Vives et al., 2000), and/or immature cortical networks of inhibitory neurons (Dealy and Tolhurst, 1974; Ahmed et al., 1997; Boudreau and Ferster, 2005).

Figure 6. A, Mean matched sampling method was used to calculate global information density for infant (red) and adult (blue) cell populations. See Results for details. B, Global information density. Scatter plot of mutual information as a function of spike count difference for all contrast pairs. Solid curve shows the fitted function for each cell group. Dashed line shows the information density for each cell group (maximum slope of the fitted function). Cross bars indicate means and SDs of mutual information and spike rate differences.

was stronger for adults $(r=-0.43, p<0.0001)$ than for infants $(r=-0.25, p<0.0007)$. The inverse correlation between $\mathrm{m}-\mathrm{FF}$ and information density was similar to the "imperfect" correlation reported for infant V1 (Rust et al., 2002).

Finally, the information density of individual neurons was negatively correlated with their dynamic range for both infants and adults (Fig. 7F). As in V1 (Rust et al., 2002), the mean (geometric) dynamic range for infant $\mathrm{V} 2$ neurons was lower (one-half) compared with that for adults. However the spread (SD) of infant dynamic range was narrower than that for adults. The inverse relationship between the information density and dynamic range was stronger for adults $(r=-0.65$, $p<0.0001)$ than for infants $(r=-0.29, p<0.0001)$. Note that many infant units with small dynamic range had relatively lower information density values, contributing to the weaker correlation.

\section{Contrast response}

The most notable difference between infants and adults was that a substantial number $(>30 \%)$ of infant units had $C_{50}$ values that exceeded $100 \%$ contrast (Fig. $3 E)$ and the average contrast versus response functions hardly saturated at $80 \%$ contrast (Fig. 3C). Also, nearly $20 \%$ of infant units had $R_{\max }$ values that exceeded 100 spikes/s (Fig. 3F). These results suggest that the contrast normalization mechanisms of many infant $\mathrm{V} 2$ neurons are not as well developed as in adults.

Our data on contrast sensitivity (1/contrast threshold) of V2 neurons parallel the behaviorally determined contrast sensitivity of macaque monkeys for 4- to 8-week-old infants and adults (Kiorpes, 2016). Specifically, the median contrast threshold of our infant monkeys was about four to five times higher than that of adults (Fig. 3D) while the contrast sensitivity of infant monkeys of comparable ages is about onefourth that of adult sensitivities (Kiorpes, 2016, their Fig. 5C). These results suggest that the contrast sensitivity of V2 neurons may exert a substantial limit on the development of contrast sensitivity in primates. 
A

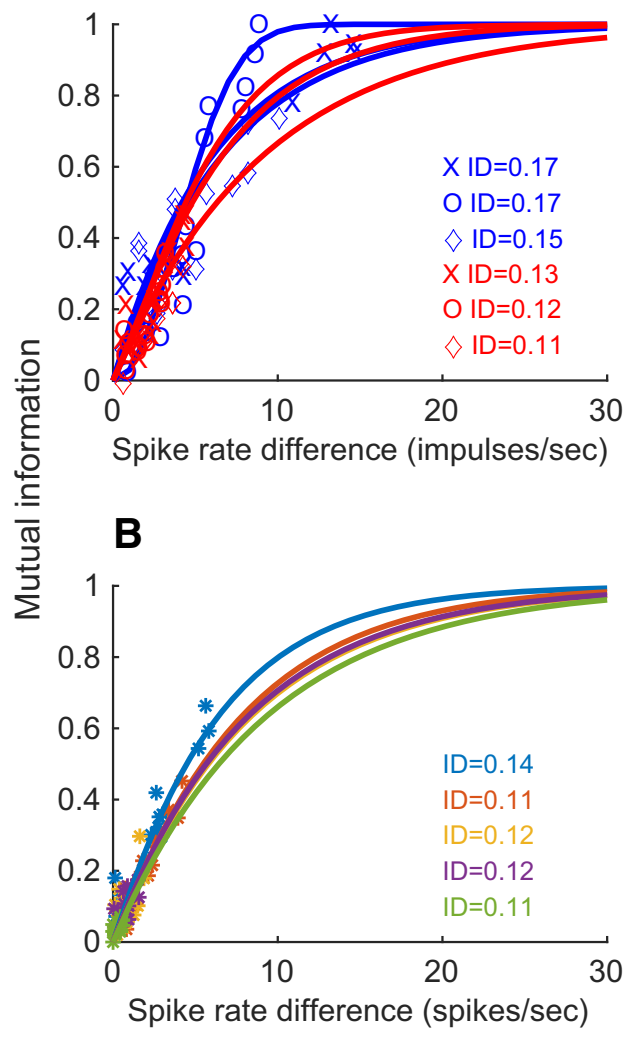

C

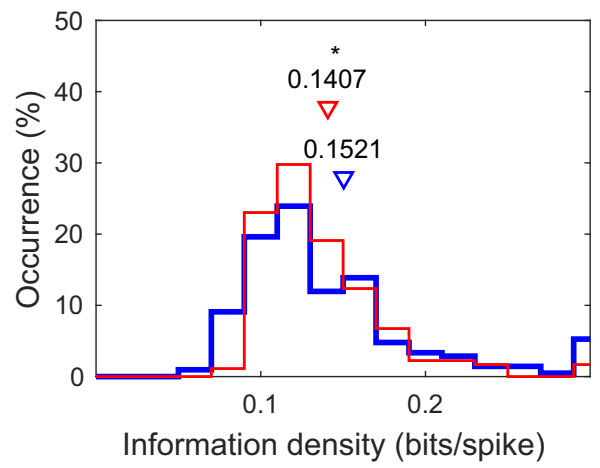

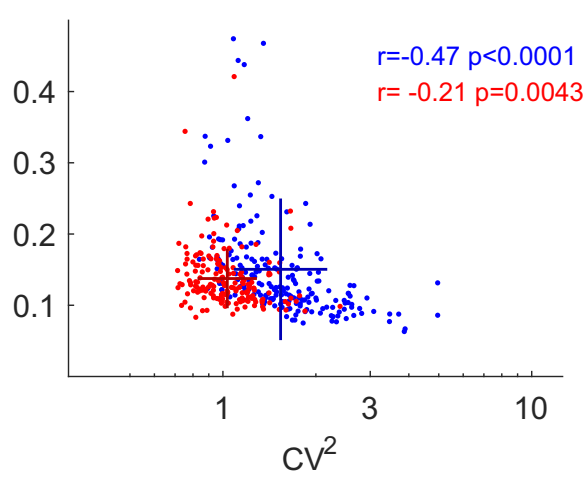

E

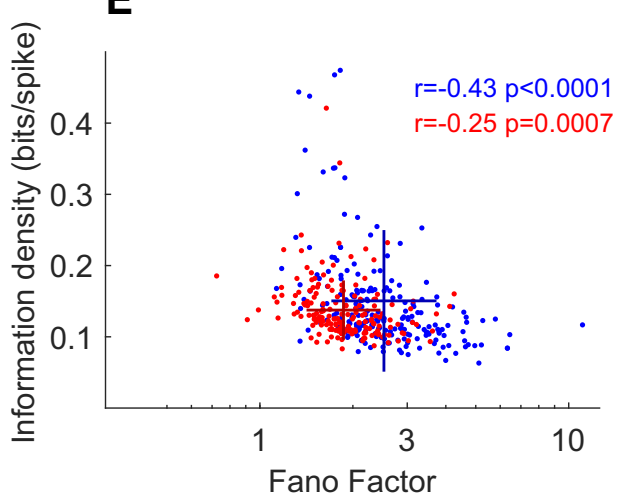

$\mathbf{F}$

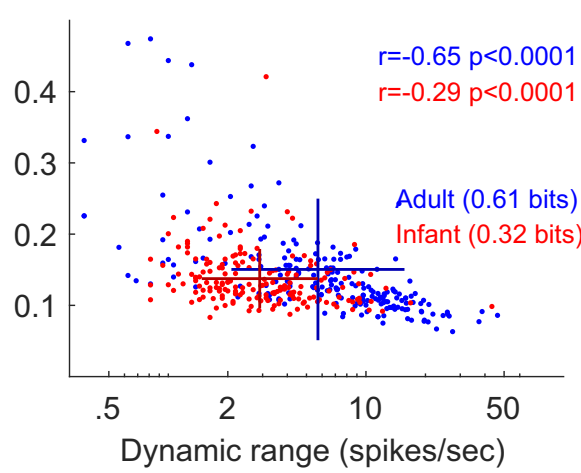

Figure 7. Information density. $\boldsymbol{A}$, Mutual information as a function of spike rate difference; for example, neurons form adults (blue) and infants (red). $\boldsymbol{B}$, Mutual information as a function of spike rate difference for neurons in Figure $4, A$ and $B$ (use colors of the functions for matching). C, Distribution of information density for adults (blue) and infants (red). Triangles indicate the mean of each cell group. ${ }^{*} p<0.05$. $\boldsymbol{D}$, Information density as a function of $\mathrm{CV}^{2}$ for each unit. Crosses indicate means and SDs for each measure. $\boldsymbol{E}$, Information density as a function of Fano factor. Crosses indicate means and SDs for each measure. $\boldsymbol{F}$, Information density as a function of dynamic range. Crosses indicate means and SDs for each measure.

Spike count variance $\left(\mathrm{CV}^{2}\right)$ and across trial variability ( $\left.\mathrm{m}-\mathrm{FF}\right)$ The spike count variance and the mean matched FF of V2 neurons in infants were much lower than those in adults regardless of stimulus contrast and also during spontaneous firing (Fig. 5D$F)$. The difference in variance of spike counts compared with that in mean spike counts played a greater role in the differences in $\mathrm{m}$-FF values. This result is similar to a previous observation in infant V1 (Rust et al., 2002). Together, as in V1, the spiking of V2 neurons in infants is far less "noisy" than in adults, predicting more reliable processing of contrast information in the visual system of infant primates. However, perceptual studies indicate otherwise (Kiorpes, 2015, 2016).

\section{Information density}

The amount of information conveyed by each action potential (information density) of infant V2 neurons was slightly but significantly less compared with that in adult V2 neurons (Figs. 6, 7). Our results are different from the observation made by a similar study in V1 (Rust et al., 2002). The apparent inconsistency between the two studies is difficult to resolve because two independent variables (infants vs adults and V1 vs V2) were not studied in the same cohort of monkeys. However, since we used the same method in one analysis to calculate the total mutual information and information density as that used in the earlier study in V1 (Fig. 7), the analysis per se is unlikely to be responsible for the 
differences between the two studies. Importantly, we also analyzed the same data using our novel approach by calculating global information density (Fig. 6), which, not surprisingly, generated a similar result.

There are a number of other reasons why the results of these two studies could be different. Most importantly, we explored V2 rather than V1. As mentioned above, the nature of input connections (and excitatory/inhibitory signals) to a single V2 neuron is quite different from that of the V1 neuron. Also, V2 neurons mature relatively later than V1 neurons (Kiorpes and Movshon, 2004; Zhang et al., 2008; Baldwin et al., 2012; Kiorpes, 2015, 2016); hence, the cortical circuits in V2 of our infant monkeys responsible for processing contrast information might not have developed as well as those in V1 for comparable postnatal ages (see below). Moreover, about half of V1 neurons in the study of Rust et al. (2002) were simple cells, whereas nearly all V2 neurons in our study were complex cells. These investigators found that simple cells in infant V1 had much higher information density ( $40-50 \%$ higher) than complex cells. Possibly, they might have encountered more simple V1 cells in infants than in adults. In our study, this was not an issue because virtually all cells were complex cells.

Importantly, anesthesia used in the two studies was substantially different (sufentanyl only for Rust et al., 2002 versus the combination of sufentanyl, propofol, and $\mathrm{N}_{2} \mathrm{O}$ for this study). Our anesthesia gave us stable recording conditions for infants, but appears to have resulted in much lower firing rates both in infants and adults. However, note that either method of anesthesia may have a larger impact on responses in V2 than in V1. Also more severe contrast adaptation in our study might have contributed to the lower response rates (Fig. 4C) and the reduced dynamic range of our V2 neurons for both infants and adults (Fig. $7 F$ ). The mean luminance of our display was much higher, the range of stimulus contrast was wider (which included $80 \%$ contrast), and the number of repeats was much higher for our study, all of which could have resulted in more severe contrast adaptation ("slow" adaptation).

In this context, it is noteworthy that a substantial number of adult V2 neurons in our study had very low firing rates $(<2.0$ spikes/s) and relatively high information density (Fig. 7F). This might have contributed to the apparent inconsistency between the two studies. However, this possibility is unlikely for a number of reasons. First, although lower dynamic range typically predicts higher information density, many infant V2 units having lower dynamic ranges in this study had relatively lower information density, instead of higher information density as observed for adults (Fig. $7 F$ ). Second, if we removed those low-firing cells (e.g., <2.0 spikes/s) from our infant and adult cell populations, information density would still be slightly but significantly higher for adults than for infants (data not shown). Finally, the calculation of global information density in this study takes large differences in firing rates between infants and adults into considerations by applying the mean matched approach, and, not surprisingly, the results remained the same (Fig. 6A).

Considered together, V2 neurons of 4- to 8-week-old infants may not convey information on stimulus contrast as well as adult monkeys, which therefore, may not "compensate" for the lower contrast sensitivity of neurons. Most importantly, our results in V2 are more consistent with perceptual data on the development of contrast sensitivity in macaque monkeys (Kiorpes, 2015, 2016).

\section{Abnormal development and spiking noise}

Experiencing unmatched images during the critical period of development leads to abnormal binocular vision and often amblyopia (for review, see Kiorpes, 2015, 2016). We previously found that the $\mathrm{CV}^{2}$ and the m-FF of V2 neurons in amblyopic monkeys are abnormally elevated, and these neural impairments are correlated with their perceptual loss (Wang et al., 2017). There are at least two possibilities to explain the emergence of noisy V2 in amblyopic monkeys; first, the spiking of V2 neurons in young infants are inherently noisy, and such immature spiking is maintained because experiencing binocular imbalance prevents normal maturation from proceeding. Alternatively, the spiking noise in V2 is adult like or even lower during early infancy. However, experiencing unmatched images during the critical period of development alters the wiring of individual V2 neurons, hence, altering the ratio of excitatory and inhibitory inputs to each neuron (Nawrot et al., 2008). These sorts of changes in the wiring of V2 could lead to enhanced noise in amblyopic visual system. Our current study in infants is more consistent with the latter possibility.

\section{Conclusion}

The firing rate, contrast sensitivity, and dynamic range of V2 neurons are substantially lower in infants than in adults. Although the spiking noise is much lower for infants compared with that for adults, the information about stimulus contrast (information density) conveyed by each spiking of V2 neurons is significantly less in infants. Together, we conclude that the combination of poor contrast sensitivity and lower information density of V2 neurons may limit the contrast sensitivity of infant primates.

\section{References}

Ahmed B, Anderson JC, Martin KA, Nelson JC (1997) Map of the synapses onto layer 4 basket cells of the primary visual cortex of the cat. J Comp Neurol 380:230-242.

Anzai A, Peng X, Van Essen DC (2007) Neurons in monkey visual area V2 encode combinations of orientations. Nat Neurosci 10:1313-1321.

Baldwin MK, Kaskan PM, Zhang B, Chino YM, Kaas JH (2012) Cortical and subcortical connections of V1 and V2 in early postnatal macaque monkeys. J Comp Neurol 520:544-569.

Blakemore C, Vital-Durand F (1981) Postnatal development of the monkey's visual system. Ciba Found Symp 86:152-171.

Boudreau CE, Ferster D (2005) Short-term depression in thalamocortical synapses of cat primary visual cortex. J Neurosci 25:7179-7190.

Carandini M, Ferster D (1997) A tonic hyperpolarization underlying contrast adaptation in cat visual cortex. Science 276:949-952.

Carandini M, Ferster D (2000) Membrane potential and firing rate in cat primary visual cortex. J Neurosci 20:470-484.

Carandini M, Heeger DJ, Senn W (2002) A synaptic explanation of suppression in visual cortex. J Neurosci 22:10053-10065.

Chino YM, Smith EL 3rd, Hatta S, Cheng H (1997) Postnatal development of binocular disparity sensitivity in neurons of the primate visual cortex. J Neurosci 17:296-307.

Churchland MM, Yu BM, Cunningham JP, Sugrue LP, Cohen MR, Corrado GS, Newsome WT, Clark AM, Hosseini P, Scott BB, Bradley DC, Smith MA, Kohn A, Movshon JA, Armstrong KM, Moore T, Chang SW, Snyder LH, Lisberger SG, Priebe NJ, et al (2010) Stimulus onset quenches neural variability: a widespread cortical phenomenon. Nat Neurosci 13:369_ 378.

Dealy RS, Tolhurst DJ (1974) Is spatial adaptation an after-effect of prolonged inhibition? J Physiol 241:261-270.

Ito M, Komatsu H (2004) Representation of angles embedded within contour stimuli in area V2 of macaque monkeys. J Neurosci 24:3313-3324.

Kiorpes L (2015) Visual development in primates: neural mechanisms and critical periods. Dev Neurobiol 75:1080-1090.

Kiorpes L (2016) The puzzle of visual development: behavior and neural limits. J Neurosci 36:11384-11393. 
Kiorpes L, Movshon JA (2004) Neural limitations on visual development. Vis Neurosci 2004:159-173.

Kiorpes L, Tang C, Hawken MJ, Movshon JA (2003) Ideal observer analysis of the development of spatial contrast sensitivity in macaque monkeys. J Vis 3:630-641.

Kohn A, Coen-Cagli R, Kanitscheider I, Pouget A (2016) Correlations and neuronal population information. Annu Rev Neurosci 39:237-256.

Martin KA, Schröder S (2013) Functional heterogeneity in neighboring neurons of cat primary visual cortex in response to both artificial and natural stimuli. J Neurosci 33:7325-7344.

Maruko I, Zhang B, Tao X, Tong J, Smith EL 3rd, Chino YM (2008) Postnatal development of disparity sensitivity in visual area 2 (V2) of macaque monkeys. J Neurophysiol 100:2486-2495.

Maunsell JH, Gibson JR (1992) Visual response latencies in striate cortex of the macaque monkey. J Neurophysiol 68:1332-1344.

Mitchell JF, Sundberg KA, Reynolds JH (2009) Spatial attention decorrelates intrinsic activity fluctuations in macaque area V4. Neuron 63:879888.

Müller JR, Metha AB, Krauskopf J, Lennie P (2001) Information conveyed by onset transients in responses of striate cortical neurons. J Neurosci 21:6978-6990.

Nawrot MP, Boucsein C, Rodriguez Molina V, Riehle A, Aertsen A, Rotter S (2008) Measurement of variability dynamics in cortical spike trains. J Neurosci Methods 169:374-390.

Rust NC, Schultz SR, Movshon JA (2002) A reciprocal relationship between reliability and responsiveness in developing visual cortical neurons. J Neurosci 22:10519-10523.

Sanchez-Vives MV, Nowak LG, McCormick DA (2000) Cellular mechanisms of long-lasting adaptation in visual cortical neurons in vitro. J Neurosci 20:4286-4299.
Shadlen MN, Newsome WT (1998) The variable discharge of cortical neurons: implications for connectivity, computation, and information coding. J Neurosci 18:3870-3896.

Tao X, Zhang B, Smith EL 3rd, Nishimoto S, Ohzawa I, Chino YM (2012) Local sensitivity to stimulus orientation and spatial frequency within the receptive fields of neurons in visual area 2 of macaque monkeys. J Neurophysiol 107:1094-1110.

Tao X, Zhang B, Shen G, Wensveen J, Smith EL 3rd, Nishimoto S, Ohzawa I, Chino YM (2014) Early monocular defocus disrupts the normal development of receptive-field structure in V2 neurons of macaque monkeys. J Neurosci 34:13840-13854.

Wang Y, Zhang B, Tao X, Wensveen JM, Smith EL Rd, Chino YM (2017) Noisy spiking in visual area V2 of amblyopic monkeys. J Neurosci 37: 922-935.

Zhang B, Zheng J, Watanabe I, Maruko I, Bi H, Smith EL 3rd, Chino Y (2005) Delayed maturation of receptive field center/surround mechanisms in V2. Proc Natl Acad Sci U S A 102:5862-5867.

Zhang B, Smith EL 3rd, Chino YM (2008) Postnatal development of onset transient responses in macaque V1 and V2 neurons. J Neurophysiol 100:1476-1487.

Zhang B, Tao X, Shen G, Smith EL 3rd, Ohzawa I, Chino YM (2013) Receptive-field subfields of V2 neurons in macaque monkeys are adultlike near birth. J Neurosci 33:2639-2649.

Zhang S, Xu M, Kamigaki T, Hoang Do JP, Chang WC, Jenvay S, Miyamichi K, Luo L, Dan Y (2014) Long-range and local circuits for top-down modulation of visual cortex processing. Science 345:660-665.

Zheng J, Zhang B, Bi H, Maruko I, Watanabe I, Nakatsuka C, Smith EL 3rd, Chino YM (2007) Development of temporal response properties and contrast sensitivity of V1 and V2 neurons in macaque monkeys. J Neurophysiol 97:3905-3916. 